\title{
Hydraulic Fracturing at Sedimentary Basin Scale
}

\author{
F. Schneider, M. Boutéca and J.P. Sarda \\ 1 Institut français du pétrole, 1 et 4, avenue de Bois-Préau, 92852 Rueil-Malmaison Cedex - France \\ email: frederic.schneider@ifp.fr
}

Résumé - Fracturation hydraulique à l'échelle des bassins sédimentaires — Le calcul du tenseur des contraintes est déterminant pour pouvoir simuler la fracturation hydraulique à l'échelle des bassins sédimentaires. Ce calcul n'est généralement pas abordé dans les modèles de bassins en raison de la complexité du problème. Pour pouvoir estimer le tenseur des contraintes, nous sommes alors amenés à formuler les hypothèses suivantes :

- une des contraintes principales est verticale et égale, en module, au poids des terrains sus-jacents ;

- la contrainte horizontale se déduit de la contrainte verticale à partir d'un coefficient de report $K_{o}$ qui est une fonction de la profondeur et du contexte géodynamique.

La consolidation est supposée être le résultat à la fois de la compaction mécanique et de la compaction chimique. La compaction mécanique est principalement due au réarrangement mécanique des grains pendant l'enfouissement. Elle peut se simuler, à l'échelle macroscopique, par une rhéologie élastoplastique. La compaction chimique est le résultat des mécanismes de pression-dissolution. Elle peut être simulée, à l'échelle macroscopique, par une rhéologie viscoplastique.

La surface de charge est complètement définie par l'union de la surface de consolidation avec les différentes limites de rupture. Cette surface de charge est alors définie par six surfaces élémentaires qui représentent la compaction verticale, la compaction horizontale, la rupture verticale en traction, la rupture horizontale en traction, la rupture subverticale en cisaillement, et la rupture subhorizontale en cisaillement.

En raison du processus de consolidation, la plupart des paramètres qui décrivent les propriétés physiques des sédiments évoluent avec l'histoire géologique. Une des difficultés est alors de quantifier le degré d'évolution du milieu poreux. Nous avons choisi ici de mesurer l'évolution des sédiments par leur porosité.

Les simulations locales ont montré que la fracturation peut apparaître dans de nombreuses configurations. Dans certaines de ces situations, le sédiment peut atteindre les limites de son domaine élastique et peut alors initier des fractures. Dans d'autres cas, le chargement du sédiment change, mais il reste sur sa surface de charge. Dans ce cas, les considérations théoriques ne sont plus valables et les critères de rupture doivent alors être définis dans le domaine plastique.

Mots-clés : fracturation hydraulique, bassin sédimentaire, perméabilité, compaction

Abstract - Hydraulic Fracturing at Sedimentary Basin Scale - One key point for simulating the hydraulic fracturing at basin scale, is to be able to compute the stress tensor. This is generally not addressed in basin model because of the complexity of this problem. In order to get access to the stress tensor we have to assume that:

- one of the principal stress is vertical and equals the overburden weight

- the horizontal stress is deduced from the vertical stress with the $\mathrm{K}_{\mathrm{o}}$ coefficient that is a function of depth and of the tectonical setting.

Consolidation is considered here as the combined effect of the mechanical compaction and the chemical compaction. The mechanical compaction is mainly caused by the rearrangement of grains during burial 
and could be represented at the macroscopical scale by an elastoplastic rheology. The chemical compaction is considered here as resulting from dissolution-precipitation mechanisms, generally induced by stress (pressure-solution). The chemical compaction could be represented at the macroscopical scale by a viscoplastic rheology.

The complete elastoplastic yield is defined by the union of the consolidation elastoplastic yield and of the different failure criteria that could be seen as elastobrittle yields. Thus, the elastoplastic yield is composed of six elementary elastoplastic yields which define the onset of vertical compaction, horizontal compaction, vertical tensile fracturing, horizontal tensile fracturing, subvertical shear fracturing, and subhorizontal shear fracturing.

Due to the consolidation, most of the parameters that describe the physical properties of the sediments evolve with the geological times. One difficulty is to quantify the degree of evolution of the porous medium during its geological history. Here, we have chosen to measure the evolution of the sediments by their porosity.

The local simulations showed that fracturing may occur is numerous configurations. Some of these configurations indicate that the sediments can reach the limit of its elastic domain and then may initiate some fracturing. In other cases, the sediment changes its loading path but stay at the elastoplastic yield surface. In this case the theoretical considerations are not valid anymore and fracture criteria should be defined in the plastic domain.

Keywords: hydraulic fracturing, sedimentary basin, permeability, compaction

\section{INTRODUCTION}

Basin modelling aims to reconstruct the accumulation of hydrocarbons at basin scale, and at geological time scale, taking into account the effects of kinematics displacements, sedimentation, erosion, compaction, temperatures history, overpressures and fluids flows (water and hydrocarbons) Furthermore, explorationists wish to address overpressure reconstruction in order to estimate the risks of drilling.

Kinematics at basin scale (faulting and/or folding) generally represents the displacements related to the tectonic stress or to the salt (or mud) tectonics. These phenomena are poorly understood, especially in term of rheology, and they are generally treated by using empirical geometrical rules (simple shear, flexural slip, etc.).

The main driving forces during hydrocarbons migration are: the pressure gradient, the gravity, and the capillary pressure gradient. The displacement of the hydrocarbons is then the result of the coupling of the conservation laws (solid, water, and hydrocarbons) with the generalised Darcy's laws and with a compaction law.

One of the goals of basin modelling is to better understand the phenomenon of "natural hydrofracturing" and its consequences at basin and reservoir scales. We want to understand and to predict the behaviour of caprock above overpressured reservoirs, fracturing correlated with in situ generated overpressure in shale, and fracturing induced by the transformation of kerogen into hydrocarbons.

The consolidation processes (mechanical compaction and chemical compaction) result from variation in the tota stress tensor, the pore pressure, the temperature and time (Schneider et al., 1996). These parameters may be derived, under certain assumptions, from the geological history of the considered basin.

The aim of basin modelling is to reconstruct the geological history of a sedimentary basin over 10 to $300 \mathrm{Ma}$. The average size of the studied area is generally in the range of $10 \mathrm{~km}$ to $100 \mathrm{~km}$ while a cell of the numerical simulator is generally $100 \mathrm{~m}$ to $1000 \mathrm{~m}$ wide and $10 \mathrm{~m}$ to $100 \mathrm{~m}$ thick. Under these conditions, there is no physical law at present that is able to simulate correctly the process of hydraulic fracturing.

There is some knowledge at laboratory scale, even if the process of hydraulic fracturing is not easy to capture. There is some more knowledge at reservoir scale where hydraulic fractures are created by local injection of fluids. In this field, one elliptical fracture is generally created from the injection point. But, the case of diffuse hydraulic fracturing due to an increase of the pore pressure (or due to a decrease of the total stress at a given pore pressure) has not been addressed from a quantitative point of view in basin modelling.

The first part of this paper is dedicated to the sediment behaviour at geological time scale. This behaviour is characterised by:

- the loading path at geological time scale;

- the constitutive law which includes the consolidation process and the rupture criteria;

- the parameters evolution related to consolidation.

The second part is dedicated to some geological applications that can be treated analytically. In particular the effect of:

- the increase of pore pressure due to generation;

- the decrease of the vertical stress due to an erosion;

- the effect of the decrease of the horizontal stress due to a tectonical event are analysed. 


\section{SEDIMENT BEHAVIOUR AT GEOLOGICAL TIME SCALE}

\subsection{Loading at Geological Time Scale}

One key point for simulating the hydraulic fracturing at basin scale, is to be able to compute the stress tensor. This is generally not addressed in basin model because of the complexity of this problem. Indeed, we need a rheology that represents the sediment behaviour at basin scale over some Ma. Then we need a model to simulate the process of hydraulic fracturing (e.g. the creation of fractures). And, at last, we need a permeability model to simulate the increase (or decrease) of permeability during the hydraulic fracturing.

In order to estimate the stress tensor all along the geological history, some assumptions should be made. The first assumption is that the total overburden weigh $\left(\sigma_{v}\right)$ is one of the main stresses. The second assumption is that the two other main stresses are horizontal and equal $\left(\sigma_{h}=\sigma_{H}\right)$.

As the vertical stress is supposed to be equal to the overburden weight, we may write:

$$
\sigma_{v}(z)=g \int_{0}^{z} \sum_{\alpha}\left(\phi_{\alpha} \rho_{\alpha}\right) \mathrm{d} z
$$

Thus, if we assume that the porosity of the sediments is given by an Athy's law:

$$
\phi=\phi^{o} \exp \left(-\frac{z}{z^{o}}\right)
$$

and that the pore space is saturated by water, the vertical stress is given by the following formula:

$$
\sigma_{v}(z)=\rho_{s} g z-\left(\rho_{s}-\rho_{w}\right) g z^{o} \phi^{o}\left(1-\exp \left(-\frac{z}{z^{o}}\right)\right)
$$

If we assume that the vertical stress $\left(\sigma_{v}\right)$ is given by the weight of the overburden then we need to have an estimate of the horizontal stress $\left(\sigma_{h}\right)$. When no measurements are available, one can:

- use empirical relationships which relate the horizontal stress to depth and overpressure such as the ones derived for the Gulf Coast by Breckels and Van Eekelen (1982);

- use estimates of the Poisson's ratio as a function of depth (e.g. Eaton, 1969) and calculating the horizontal stress by the following formula (e.g. Magara, 1983):

$$
\sigma_{h}^{\prime}=\frac{v}{1-v} \sigma_{v}^{\prime}
$$

which use is justified in the elastic domain when there is no horizontal deformation;

- assume that the total horizontal stress can be deduced from the total vertical stress by the following formula (e.g. Schneider et al., 1997):

$$
\sigma_{h}=K_{o} \sigma_{v}
$$

This third formulation get the advantage to be independent of the knowledge of the sediment rheology. Indeed this is the sediment rheology which imposes the definition of the effective stress. Ko may be a function of depth (e.g. Breckels and Van Eekelen, 1982; Grauls, 1996) for a given basin. According to the tectonical environment, Ko may vary from values lower than 1 in distensive area to values greater than 1 in compressive area. For example, from values given by Grauls (1996), for passive margins (type I) we may derive the following expression:

$$
K_{o}(z)=0.85-0.18 \exp \left(-\frac{z}{2650}\right)
$$

Under the following assumptions:

- one of the principal stress is vertical and equals the overburden weight;

- the horizontal stress is deduced from the vertical stress with the $K_{0}$ coefficient;

- the pore pressure is normal and hydrostatic;

- the loading is monotonous; the evolution of a sediment during its geological history (type I) is represented in the $\left(Q, P^{\prime}\right)$ diagram (Fig. 1) by a curve characterised by an increase of the mean effective stress and an increase of the deviatoric stress with the increasing depth.

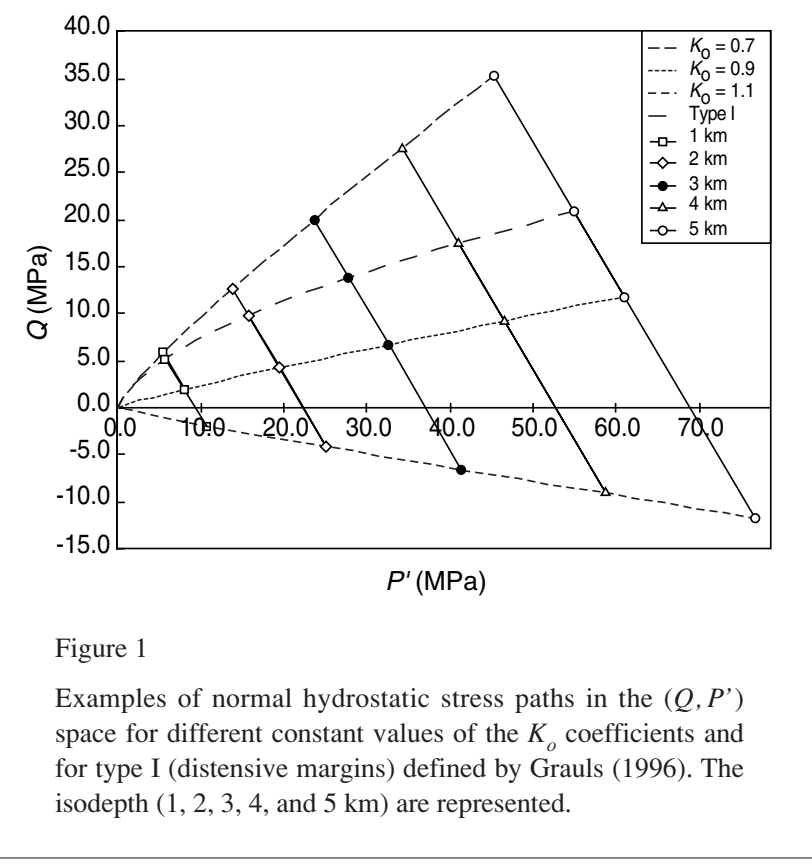

\subsection{Constitutive Law}

Since we are working on the fracturing of sediments at geological time scale, it appears necessary to trace out the sediment evolution during its history. From the top to the bottom of the sedimentary column we distinguish four major 
horizons (Schneider et al., 1994). The first horizon at the top of the sedimentary column, is made up of muds. Beneath this horizon we consider that the sediment forms a soil having a thickness of some meters. The appearance of cemented grains marks the start of the third horizon. It is made of the sedimentary rock stricto sensu. The end of this third horizon and the beginning of the fourth one is marked by the beginning of the metamorphism.

The previous geometrical description of the sedimentary column could be considered as an historical evolution of sediments, that are muds when they are young, then they become soils, sedimentary rocks and metamorphic rocks.

Determining all the mechanisms that cause this evolution would appear to be impossible. However, attempting to determine the major physical phenomena in the history of the sediment is feasible. This operation has already been tackled by Hedberg (1936). Here we will only consider the sedimentary rock stricto sensu, and will use the word consolidation to represent all the physical processes that act on the sediment during its geological history.

Consolidation is considered here as the combined effect of the mechanical compaction and the chemical compaction (Schneider et al. 1996).

The mechanical compaction is mainly caused by the rearrangement of grains during burial. This mechanism is efficient near the surface. The mechanical compaction could be represented at the macroscopical scale by an elastoplastic rheology (Schneider et al., 1996).

The chemical compaction is considered here as resulting from dissolution-precipitation mechanisms, generally induced by stress (pressure-solution). This mechanism generally becomes to be active at a depth of a few hundred meters for carbonate sediments and about one to $2 \mathrm{~km}$ for sandstones. The chemical compaction could be represented at the macroscopical scale by a viscoplastic rheology (Schneider et al., 1996).

The resulting elasto-viscoplastic rheology could be written as follows (from Schneider et al., 1996):

$$
\frac{\mathrm{d} \phi}{\mathrm{d} t}=-\beta\left(P^{\prime}\right) \frac{\mathrm{d} P^{\prime}}{\mathrm{d} t}-\alpha(\phi, T) P^{\prime}
$$

At a given time, the consolidation elastoplastic yield is supposed to be given in the $\left(Q, P^{\prime}\right)$ space by the classical following equation (e.g. Longuemare, 1996):

$$
\mathrm{Q}^{2}+P^{\prime 2}-P_{c}^{\prime 2}=0
$$

where $P^{\prime}$ is the consolidation mean effective stress which evolves with the geological history of the sediment.

According to many authors, different failure criteria should be considered. Here we will consider tensile fracturing and shear fracturing.
The tensile vertical failure criterion is:

$$
\sigma_{h}-p=-T
$$

where $T$ is the tensile strength $(T>0)$.

In the $\left(Q, P^{\prime}\right)$ space, this criterion becomes:

$$
Q=3\left(T+P^{\prime}\right)
$$

The tensile horizontal failure criterion is:

$$
\sigma_{v}-p=-T
$$

In the $\left(Q, P^{\prime}\right)$ space, this criterion becomes:

$$
Q=-3 / 2\left(T+P^{\prime}\right)
$$

It should be noticed that the tensile criteria could be improved by adding a transition toward shear fracturing as it is done in the Griffith criterion.

For shear fracturing we have chosen the Mohr criterion but other criteria could be used instead. The Mohr criterion is given by:

$$
\tau=c+\tan (\varphi) \sigma_{n}
$$

where $c$ is the cohesion and $\varphi$ the friction angle. The rupture angle $\theta$ is given by:

$$
2 \theta=\varphi+\pi / 2
$$

The rupture criterion could be written as follows:

$$
\frac{\sigma_{v}^{\prime}-\sigma_{h}^{\prime}}{2}=c \cos (\varphi)+\frac{\sigma_{v}^{\prime}+\sigma_{h}^{\prime}}{2} \sin (\varphi)
$$

In the $\left(Q, P^{\prime}\right)$ space, this criterion becomes:

$$
\begin{cases}\text { if } \sigma_{v}>\sigma_{h} & Q=+\frac{6 \sin (\varphi)}{3-\sin (\varphi)}\left(P^{\prime}+c \cot (\varphi)\right) \\ \text { if } \sigma_{v}<\sigma_{h} & Q=-\frac{6 \sin (\varphi)}{3+\sin (\varphi)}\left(P^{\prime}+c \cot (\varphi)\right)\end{cases}
$$

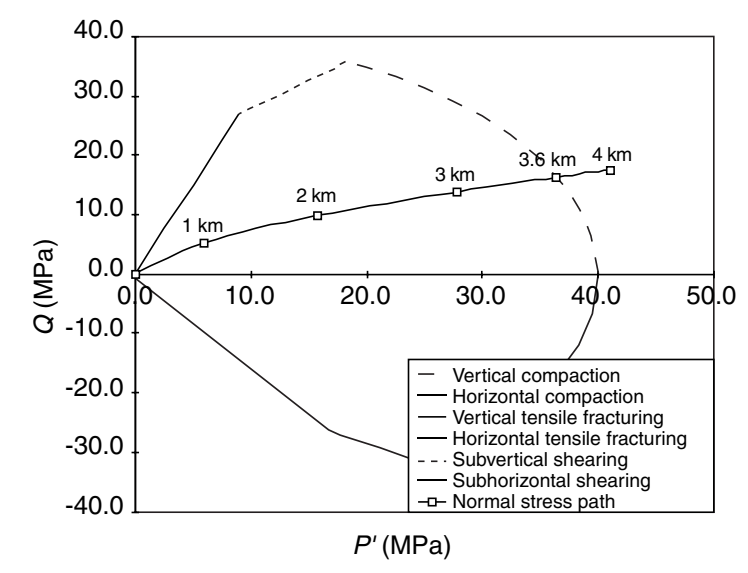

Figure 2

Example of normal hydrostatic stress path for a sediment from deposition to 4 th $\mathrm{km}$ depth. The complete elastoplastic yield (see text) is drawn for the sediment at $3.6 \mathrm{~km}$ depth. 
The complete elastoplastic yield is defined (Fig. 2) by the union of the consolidation elastoplastic yield and of the different failure criteria that could be seen as elastobrittle yields. Thus, the elastoplastic yield is composed of six elementary elastoplastic yields which define the onset of vertical compaction, horizontal compaction, vertical tensile fracturing, horizontal tensile fracturing, subvertical shear fracturing, and subhorizontal shear fracturing.

\subsection{Parameters Evolution with Consolidation}

Due to the consolidation (cumulative effect of the mechanical compaction and the chemical compaction), most of the parameters that describe the physical properties of the sediments evolve with the geological times. One difficulty is to quantify the degree of evolution of the porous medium during its geological history. Here, we have chosen to measure the evolution of the sediments by their porosity. This choice is generally done by the authors because porosity is one of the physical properties that can be measured easily. But in case of strong diagenesis with creation of high secondary porosity, this choice could become problematic.

The elastoplastic coefficient is given by (Schneider et al., 1996):

$$
\beta\left(P^{\prime}\right)=\frac{\phi_{a}}{E_{a}} \exp \left(-\frac{P^{\prime}}{E_{a}}\right)+\frac{\phi_{b}}{E_{b}} \exp \left(-\frac{P^{\prime}}{E_{b}}\right)
$$

where $\phi_{a}, E_{a}, \phi_{b}, E_{b}$ are coefficients which characterise the sediment. These coefficients may be calibrated by using observed porosity-depth data from sediment that have not suffer diagenesis or using experimental laboratory measurements (Schneider et al., 1996).

The viscoplastic coefficient is given by (Schneider et al., 1996)

$$
\alpha(\phi, T)=\frac{1-\phi}{\mu_{s}^{o} \exp \left(\frac{E}{R}\left(\frac{1}{T}-\frac{1}{T^{o}}\right)\right)}
$$

where $\mu^{o}$, is the macroscopical sediment viscosity at $T^{o}$, and $E$ is the activation energy. These coefficients may be calibrated from set of observed data (Schneider et al., 1996).

The Young's modulus could be expressed as a function of the porosity. For example, for chalk, Engstrom (1992) proposed the following relation:

$$
E=224.8 \exp (-11.2 \phi)(\mathrm{GPa})
$$

The Poisson's coefficient could be expressed as a function of the porosity. For example, for chalk, Engstrom (1992) proposed the following relation:

$$
v=0.117+0.21 \phi
$$

Other data provided for limestones by Boutéca and Sarda (1995) show that the Poisson's coefficient may decrease with the increasing porosity.

In the plastic domain we suppose that the effective stress coefficient is equal to 1 . In the elastic domain, the Biot coefficient could be expressed as a function of the porosity (Schneider et al., 1993, after Boutéca et al., 1991):

$$
b(\phi)=\frac{\phi}{\phi+a\left(\phi^{0}-\phi\right)}
$$

The tensile strength of sediment is generally weak. For example Table 1 gives some ranges extracted from Lama and Vutukuri (1978). If we assume that the sediment is fractured, the tensile strength could decrease to 0 . Indeed, the criterion for the opening of existing fractures is the same we wrote for a tensile fracture but with $T=0$.

TABLE 1

Range of values of the tensile strength for different type of rocks

\begin{tabular}{l|l} 
Sandstones & $T=2$ to $6 \mathrm{MPa}$ \\
Salt & $T=1$ to $3 \mathrm{MPa}$ \\
Chalk & $T=1$ to $4 \mathrm{MPa}$ \\
Shale & $T=1$ to $3 \mathrm{MPa}$ \\
\hline
\end{tabular}

The cohesion of sediment is generally known to increase with depth or to increase as the porosity decreases. The cohesion $c$ could be expressed as a function of the porosity and mineral content. For example, for chalk we can use the empirical relation (Rhett et al., 1992):

$$
c=20.9-41.1 \phi(\mathrm{MPa})
$$

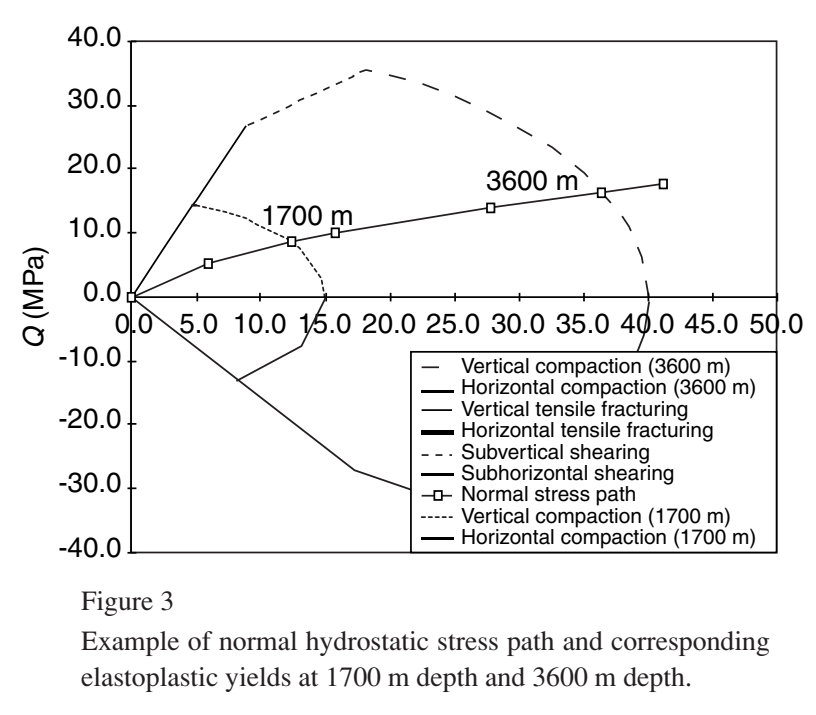


Generally the friction angle increases with depth. Furthermore, the friction angle could be expressed as a function of the porosity and mineral content. For example, for chalk we can use (Rhett et al., 1992):

$$
\varphi=56.1-105.6 \phi\left(^{\circ}\right)
$$

Once the expression of the coefficients as functions of the porosity are given, the evolution of the different elastoplastic yield surfaces can be drawn in the $\left(Q, P^{\prime}\right)$ space. This has been done in Figure 3 in which the yield surfaces have been drawn for chalk at $1.7 \mathrm{~km}$ and $3.6 \mathrm{~km}$. In this figure, itshould be noticed that at $1.7 \mathrm{~km}$ depth, the elastic domain is defined by the tensile failure criteria and the consolidation yield surface while the elastic domain needs all the rupture criteria to be defined at $3.6 \mathrm{~km}$

\section{APPLICATIONS TO GEOLOGICAL CASE} WITH LOCAL MODELLING

The sediment is supposed to be at its elastoplastic boundary. An increase of the pore pressure, a decrease of the total vertical stress or a decrease of the horizontal stress generally move the porous medium through its elastic domain. In this case, we can use the following equations (Coussy, 1991):

$$
\begin{aligned}
\sigma_{i j}-\sigma_{i j}^{0} & =\left(K-\frac{2 \mu}{3}\right) \operatorname{tr} \varepsilon \delta_{i j}+2 \mu \varepsilon_{i j}+b\left(p-p_{0}\right) \delta_{i j} \\
p-p_{0} & =M\left(b \operatorname{tr} \varepsilon+\frac{m-m_{0}}{\rho_{f}^{0}}\right)
\end{aligned}
$$

Written in principal axis and assuming isotropic stresses in the horizontal plan, the previous system becomes:

$$
\begin{aligned}
\sigma_{v}-\sigma_{v}^{0} & =\left(K-\frac{2 \mu}{3}\right)\left(\varepsilon_{v}+2 \varepsilon_{h}\right)+2 \mu \varepsilon_{v}+b\left(p-p_{0}\right) \\
\sigma_{h}-\sigma_{h}^{0} & =\left(K-\frac{2 \mu}{3}\right)\left(\varepsilon_{v}+2 \varepsilon_{h}\right)+2 \mu \varepsilon_{h}+b\left(p-p_{0}\right) \\
p-p_{0} & =M\left(\mathrm{~b}\left(\varepsilon_{v}+2 \varepsilon_{h}\right)+\frac{m-m_{0}}{\rho_{f}^{0}}\right)
\end{aligned}
$$

For the numerical applications we used the parameters given in Table 2 which are average values for carbonates extracted from Rhett et al. (1994), Engstrom (1994), Boutéca and Sarda (1995).

TABLE 2

Average values used for the numerical applications

\begin{tabular}{c|c|c|c|c|c|c|c|c}
\hline$P^{\prime} c(\mathrm{MPa})$ & $c(\mathrm{MPa})$ & $\varphi$ & $T$ & $b$ & $v$ & $M(\mathrm{GPa})$ & $K(\mathrm{GPa})$ & $\mu(\mathrm{MPa})$ \\
\hline 40 & 10 & 25 & 0 & 0.3 & 0.3 & 40 & 30 & 14 \\
\hline
\end{tabular}

\subsection{Increase of Pore Pressure (Generation)}

Here $p$ is imposed and we will examine the evolutions of the vertical and the horizontal effective stress.

\subsubsection{Constant Vertical Stress}

\section{and Constant Horizontal Stress}

The assumption is expressed as: $\sigma_{v}=\sigma_{v}^{0}$ and $\sigma_{h}=\sigma_{h}^{0}$. Thus, the increase of pore pressure will induce a translation equal to the pore pressure increase, of the Mohr circle towards the vertical axis. In this case we can have a tensile vertical fracture or a shear fracture depending on which criterion is first reached.

$$
\begin{aligned}
\delta Q & =0 \\
\delta P^{\prime} & =-\delta p \\
\frac{\delta Q}{\delta P^{\prime}} & =0
\end{aligned}
$$

In the $\left(Q, P^{\prime}\right)$ diagram (Fig. 4), the point which represents the state of the considered sediment at $3.6 \mathrm{~km}$ will go horizontally toward the $Q$ axis and may reach the vertical tensile fracturing criterion.

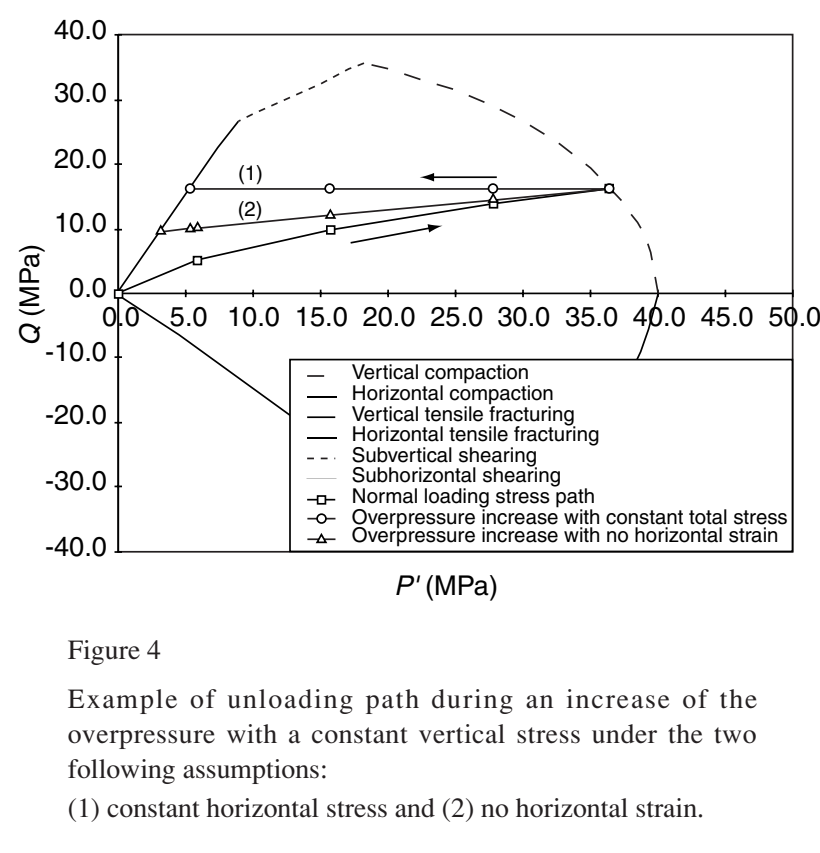

\subsubsection{Constant Vertical Stress and No Horizontal Strain}

The assumptions are: $\sigma_{v}=\sigma^{o}$, and $\varepsilon_{h}=0$. Under these hypotheses, the system becomes:

$$
\begin{aligned}
0 & =\left(K-\frac{2 \mu}{3}\right)\left(\varepsilon_{v}+0\right)+2 \mu \varepsilon_{v}+b\left(p-p_{0}\right) \\
\sigma_{h}-\sigma_{h}^{0} & =\left(K-\frac{2 \mu}{3}\right)\left(\varepsilon_{v}+0\right)+0+b\left(p-p_{0}\right) \\
p-p_{0} & =M\left(b\left(\varepsilon_{v}+0\right)+\frac{m-m_{0}}{\rho_{f}^{0}}\right)
\end{aligned}
$$


We derive from this system:

$$
\begin{aligned}
\varepsilon_{v} & =-\frac{b}{K_{0}+\frac{4 \mu}{3}}\left(p-p_{0}\right) \\
\sigma_{h}-\sigma_{h}^{0} & =\beta\left(p-p_{0}\right) \text { with } \beta=b \frac{1-2 v}{1-v}
\end{aligned}
$$

Thus, if the pore pressure increases, there is a vertical dilatation of the porous medium and an increase of the horizontal stress if $\beta$ is different from 0 . $\beta$ is equal to 0 when the Biot coefficient $(b)$ is equal to 0 or when the Poisson ratio coefficient $(v)$ is equal to 0.5 . Generally $\beta$ is greater than 0 and the evolution of the Mohr circle, when the pore pressure increases, is a translation toward the origin and a decrease of the radius.

In the $\left(Q, P^{\prime}\right)$ space we can write:

$$
\begin{aligned}
\delta Q & =-\beta \delta p \\
\delta P^{\prime} & =-\left(1-\frac{2}{3} \beta\right) \delta p \\
\frac{\delta Q}{\delta P^{\prime}} & =\frac{\beta}{1-\frac{2}{3} \beta}
\end{aligned}
$$

It should be noted that $P^{\prime}$ is defined as the Terzaghi effective stress. This is not the Biot effective stress except when $b=1$.

According to the original position and according to the properties, the sediment can reach different rupture criteria. For instance, in the example shown in Figure 4, at $3.6 \mathrm{~km}$ depth, the hydraulic fractures will be vertical.

\subsection{Decrease of the Vertical Stress (Erosion)}

2.2.1 Constant Water Pressure and No Horizontal Strain

This local simulation may represent the erosion of a partially open system with constant pore pressure and no lateral strain. The assumptions are written as:

$$
p=p^{0} \text { and } \varepsilon_{h}=0
$$

Under these hypotheses, the system gives:

$$
\begin{aligned}
& \sigma_{v}-\sigma_{v}^{0}=\left(K-\frac{2 \mu}{3}\right)\left(\varepsilon_{v}+0\right)+2 \mu \varepsilon_{v}+b(0) \\
& \sigma_{h}-\sigma_{h}^{0}=\left(K-\frac{2 \mu}{3}\right)\left(\varepsilon_{v}+0\right)+0+b(0) \\
& 0=M\left(b\left(\varepsilon_{v}+0\right)+\frac{m-m_{0}}{\rho_{f}^{0}}\right) \\
& \delta \sigma_{h}=\frac{\lambda}{\lambda+2 \mu} \delta \sigma_{v}=\frac{v}{1-v} \delta \sigma_{v}
\end{aligned}
$$

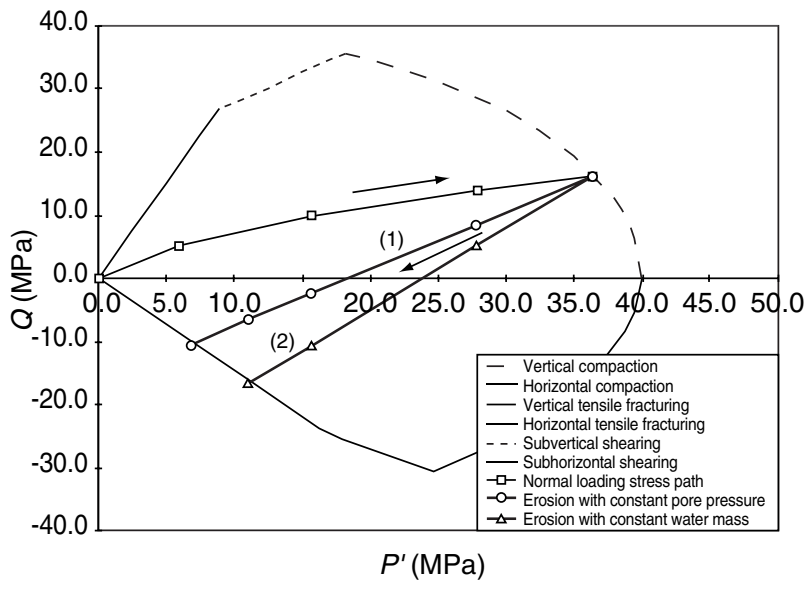

Figure 5

Example of unloading path during decrease of the vertical stress (erosion) with no horizontal strain and under the two following assumptions:

(1) constant pore pressure and (2) constant water mass.

In the $\left(Q, P^{\prime}\right)$ space we can write:

$$
\begin{aligned}
\delta Q & =\frac{1-2 v}{1-v} \delta \sigma_{v} \\
\delta P^{\prime} & =\frac{1}{3} \frac{1+v}{1-v} \delta \sigma_{v} \\
\frac{\delta Q}{\delta P^{\prime}} & =3 \frac{1-2 v}{1+v}
\end{aligned}
$$

The $\left(Q, P^{\prime}\right)$ space given in Figure 5 , shows that at $3.6 \mathrm{~km}$ depth, the fractures which are generated are horizontal (tensile horizontal fracturing).

\subsubsection{Constant Water Mass and No Horizontal Strain}

This local simulation may represent an erosion of a closed system with no lateral strain. The assumptions are written as:

$$
m=m^{0} \text { and } \varepsilon_{h}=0
$$

Under these hypotheses, the system becomes:

$$
\begin{aligned}
\sigma_{v}-\sigma_{v}^{0} & =\left(K_{0}-\frac{2 \mu}{3}\right)\left(\varepsilon_{v}+0\right)+2 \mu \varepsilon_{v}+b\left(p-p_{0}\right) \\
\sigma_{h}-\sigma_{h}^{0} & =\left(K_{0}-\frac{2 \mu}{3}\right)\left(\varepsilon_{v}+0\right)+0+b\left(p-p_{0}\right) \\
p-p_{0} & =M\left(b\left(\varepsilon_{v}+0\right)+0\right)
\end{aligned}
$$

This can be written in the following form:

$$
\begin{aligned}
\delta \sigma_{h} & =\frac{\lambda+M b^{2}}{\lambda+2 \mu+M b^{2}} \delta \sigma_{v} \\
\delta p & =\frac{M b}{\lambda+2 \mu+M b^{2}} \delta \sigma_{v}
\end{aligned}
$$


In the $\left(Q, P^{\prime}\right)$ space we can write:

$$
\begin{aligned}
\delta Q & =\frac{2 \mu}{\lambda+2 \mu+M b^{2}} \delta \sigma_{v} \\
\delta P^{\prime} & =\frac{K_{o}-M b(1-b)}{\lambda+2 \mu+M b^{2}} \delta \sigma_{v} \\
\frac{\delta Q}{\delta P^{\prime}} & =\frac{2 \mu}{K_{o}-M b(1-b)}
\end{aligned}
$$

In Figure 5, it can be seen that the conclusions concerning the fracturing are the same we had for the previous example for the point representing a sediment at $3.6 \mathrm{~km}$ depth. In this case the path reaches the tensile horizontal fracturing criterion.

\subsection{Decrease of the Horizontal Stress (Tectonics)}

2.3.1 Constant Water Pressure and Constant Vertical Stress

In this case, we simulate a superimposition of a distensive horizontal tectonic event in a system where the pore pressure and the overburden weight are kept constant. These assumptions are written as: $p=p^{0}$ and $\sigma_{v}=\sigma_{v}^{0}$.

Under these hypotheses, the system gives:

$$
\begin{aligned}
0 & =\left(K-\frac{2 \mu}{3}\right)\left(\varepsilon_{v}+2 \varepsilon_{h}\right)+2 \mu \varepsilon_{v}+0 \\
\sigma_{h}-\sigma_{h}^{0} & =\left(K-\frac{2 \mu}{3}\right)\left(\varepsilon_{v}+2 \varepsilon_{h}\right)+2 \mu \varepsilon_{h}+0
\end{aligned}
$$

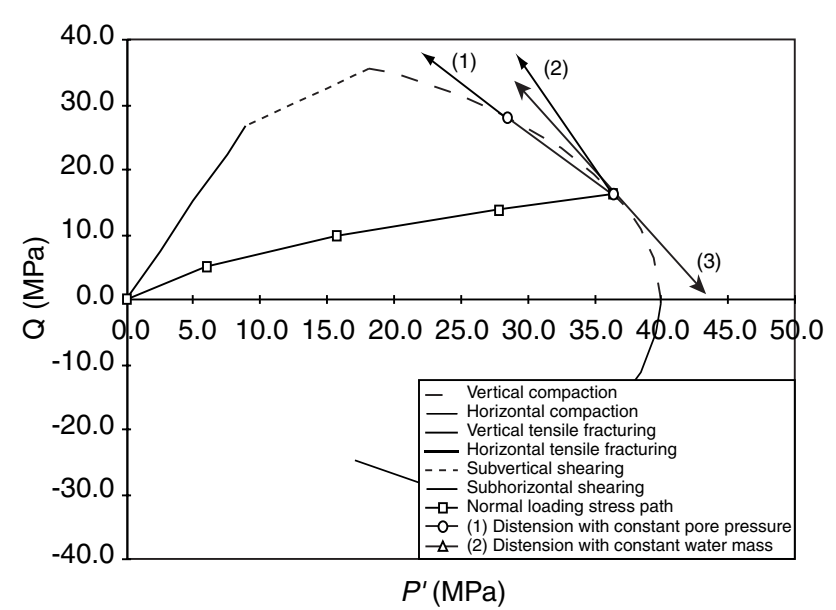

Figure 6

Example of unloading path during decrease of the horizontal stress (distension) with no horizontal strain and under the two following assumptions:

(1) constant pore pressure and (2) constant water mass. (3) is the tangent to the vertical compaction yield surface at the considered point.
And, in the $\left(Q, P^{\prime}\right)$ space we can write:

$$
\begin{aligned}
\delta Q & =-\delta \sigma_{h} \\
\delta P^{\prime} & =\frac{2}{3} \delta \sigma_{h} \\
\frac{\delta Q}{\delta P^{\prime}} & =-\frac{3}{2}
\end{aligned}
$$

The results presented in Figure 6 show that for a sediment at $3.6 \mathrm{~km}$ depth, the criterion reached is the vertical compaction one. Thus, during the distension, the sediment stops to compact and go toward its elastic domain, then it reaches its vertical compaction yield.

\subsubsection{Constant Water Mass and Constant Vertical Stress}

In this case, we simulate a superimposition of a distensive horizontal tectonic event in a closed system in which the overburden weight is kept constant. These assumptions are written as:

$$
m=m^{0} \text { and } \sigma_{v}=\sigma_{\mathrm{v}}^{0}
$$

Under these hypotheses, the system becomes:

$$
\begin{aligned}
0 & =\left(K_{0}-\frac{2 \mu}{3}\right)\left(\varepsilon_{v}+2 \varepsilon_{h}\right)+2 \mu \varepsilon_{v}+b\left(p-p_{0}\right) \\
\sigma_{h}-\sigma_{h}^{0} & =\left(K_{0}-\frac{2 \mu}{3}\right)\left(\varepsilon_{v}+2 \varepsilon_{h}\right)+2 \mu \varepsilon_{h}+b\left(p-p_{0}\right) \\
p-p_{0} & =M\left(b\left(\varepsilon_{v}+2 \varepsilon_{h}\right)+0\right)
\end{aligned}
$$

By summing the first line with two times the second one, we derive the following system:

$$
\begin{aligned}
2 \delta \sigma_{h} & =3\left(K_{0}-\frac{2 \mu}{3}\right) \varepsilon+2 \mu \varepsilon+3 b \delta p=3 K_{0} \varepsilon+3 b \delta p \\
\varepsilon & =\frac{1}{M b} \delta p
\end{aligned}
$$

Thus we obtain:

$$
\delta \sigma_{h}=\frac{3}{2} \frac{K_{0}+M b^{2}}{M b} \delta p
$$

In the $\left(Q, P^{\prime}\right)$ space we can write:

$$
\begin{aligned}
\delta Q & =-\frac{3}{2} \frac{K_{0}+M b^{2}}{M b} \delta p=-\delta \sigma_{h} \\
\delta P^{\prime} & =\frac{K_{0}-M b(1-b)}{M b} \delta p=\frac{2}{3} \frac{K_{0}-M b(1-b)}{K_{0}+M b^{2}} \delta \sigma_{h} \\
\frac{\delta Q}{\delta P^{\prime}} & =-\frac{3}{2} \frac{K_{0}+M b^{2}}{K_{0}-M b(1-b)}
\end{aligned}
$$

In this case, the previous theoretical considerations are not valid anymore. Indeed the calculated stress path in the elastic domain are not valid and should be calculated in the plastic domain. Only a complete simulation will allow us to conclude in this case. 


\section{CONCLUSIONS}

The fracturing of a sediment is mainly controlled by the state of the porous medium (consolidation and diagenesis) and by the loading path. The evolution of the rock properties at geological time scale is poorly documented. These evolutions could be simulated by using empirical parameters/porosity relationships. The estimation of the loading path is only accessible through basin simulations but no basin simulators are able to account for 3D consolidation and diagenesis at basin scale and at geological time scale.

The local simulations showed that fracturing may occur is numerous configurations. Some of these configurations indicate that the sediments can reach the limit of its elastic domain and then may initiate some fracturing. In other cases, the sediment changes its loading path but stay at the elastoplastic yield surface. In this case the theoretical considerations are not valid anymore and fracture criteria should be defined in the plastic domain.

The model presented here has been illustrated with data relevant to chalk and carbonates. Nevertheless, it is general and could be applied to other sediments, such as sandstones, siltstones and shale since we are able to define the lithological dependent parameters.

Due to the number of different configuration for initiating fracturing in sediments, it seems that only the study of specific cases extracted from real cases would give realistic insight into the general process of hydraulic fracturing.

\section{ACKNOWLEDGMENTS}

We would like to thank Statoil who partially financed this work through IFP agreement No. 20.306 (Statoil agreement No. T-123.632).

\section{REFERENCES}

Boutéca, M. and Sarda, J.P. (1995) Experimental Measurements of Thermopor Media, Charlez, P. (ed.) A.A. Balkema, Rotterdam, Brookfield.
Boutéca, M., Sarda, J.P. and Laurent, J. (1991) Rock Mechanics Contribution to the Determination of Fluid Flow Properties Second European Core Analysis Symposium (Eurocas II), London, 20-22 May.

Breckels, I.M. and van Eekelen, H.A.M. (1982) Relationship between Horizontal Stress and Depth in Sedimentary Basins. J. Petrol. Technol., 34, 2191-2199.

Coussy, O. (1991) Mécanique des milieux poreux, Éditions Technip, Paris.

Eaton, B.A. (1969) Fracture Gradient Prediction and its Application in Oil Field Operations. J. Petrol. Technol., 21, 1353-1360.

Engstrom, F. (1992) Rock Mechanical Properties of Danish North Sea Chalk. Fourth North Sea Chalk Symposium, Deauville.

Hedberg, H.D. (1936) Gravitational Compaction of Clays and Shales. American Journal of Science, 31, 241-287.

Grauls, D. (1996) Minimum Principal Stress as a Control of Overpressures in Sedimentary Basins. Proceeding of the 8th Conference on Exploration and Production. IFP, RueilMalmaison, 9-10 December. IFP Report No. 43313.

Lama, R.D. and Vutukuri, V.S. (1978) Handbook on Mechanical Properties of Rocks. Trans Tech Publications.

Longuemare, P. (1996) Couplage hydromécanique pour des lois de comportement non linéaire. Applications à des problème pétroliers. Thesis, University of Science and Technology, Lille. Magara, K. (1983) Physical Constraints of Faulting in a Geopressured Sedimentary Basin. J. Petrol. Geol., 5, 417-426.

Rhett, D.W. and Teufel, L.W. (1992) Failure Criteria for High Porosity North Sea Chalk. Fourth North Sea Chalk Symposium Deauville.

Schneider, F., Burrus, J. and Wolf, S. (1993) Modelling Overpressures by Effective-Stress/Porosity Relationships in LowPermeability Rocks: Empirical Artifice or Physical Reality? Basin Modelling: Advances and Applications, Doré, A.G. et al (eds.), NPF Special Publication, 3, 333-341, Elsevier, Amsterdam. Schneider, F., Potdevin, J.L., Wolf, S.and Faille, I. (1994) Modèle de compaction élastoplastique et viscoplastique pour simulateur de bassins sédimentaires. Revue de l'Institut français du pétrole. 49, 2, 1-8

Schneider, F., Potdevin, J.L., Wolf, S. and Faille, I. (1996) Mechanical and Chemical Compaction Model for Sedimentary Basin Simulator. Tectonophysics, 263, 307-317.

Schneider, F., Boutéca, M., Sarda, J.P., Longuemare, P., Vincké, O. Faille, I. and Wolf, S. (1997) Hydrofrac 1996 Annual Report Part 1: Theory. IFP Report No. 43551. 


\section{APPENDIX}

\section{Notations and Definitions}

We assume that compression and shortening are positive. $\overline{\bar{\sigma}}=\left(\sigma_{i j}\right)$ is the stress tensor, here we assume that:

$$
\overline{\bar{\sigma}}=\left(\begin{array}{ccc}
\sigma_{h} & 0 & 0 \\
0 & \sigma_{h} & 0 \\
0 & 0 & \sigma_{v}
\end{array}\right)
$$

$P=\frac{1}{3} I_{1}=\frac{1}{3}\left(\sigma_{v}+2 \sigma_{h}\right)$ is the mean stress

$P^{\prime}=P-p$

is the mean effective stress

$\overline{\bar{s}}=\overline{\bar{\sigma}}-P \overline{\overline{1}}$

is the deviatoric stress tensor.

$$
Q=\sqrt{3 J_{2}}=\sqrt{\frac{3}{2} \operatorname{tr}\left(s^{2}\right)}=\left|\sigma_{v}-\sigma_{h}\right|
$$

is the deviatoric stress. Here we take $Q=\sigma_{v}-\sigma_{h}$.

\section{Relations}

From the definitions of $P$ and $Q$ we can derive the following expressions:

$$
\sigma_{v}=P+\frac{2}{3} Q \quad \text { and } \quad \sigma_{h}=P-\frac{1}{3} Q
$$

We have the classical relations:

$$
\begin{gathered}
\lambda=K-\frac{2 \mu}{3}=\frac{E v}{(1+v)(1-2 v)} \\
\mu=\frac{E}{2(1+v)} \quad K=\frac{E}{3(1-2 v)}
\end{gathered}
$$

